\title{
1 An open-source method for analysis of confocal calcium 2 imaging with sparse cells \\ 3
}

4 Alisa A. Omelchenko ${ }^{1}$, Lina $\mathrm{Ni}^{1^{*}}$

$5{ }^{1}$ School of Neuroscience, Virginia Tech, Blacksburg, Virginia, United States of America, 24061

6

$7 \quad{ }^{*}$ Corresponding author

8 E-mail: linani@vt.edu (LN)

9 Keywords: Calcium imaging, Confocal microscopy, TrackMate, Fiji, Single-cell resolution 


\section{Highlights:}

11 - TrackMate is applied to analyze calcium changes at single-cell resolution.

12 - This method depends on an open-source Fiji software, ensures reproducibility, and

13 requires no coding ability.

14 - Practice images and a step-by-step guide are provided to implement this method in

$15 \quad$ various cell types and animal models. 


\section{Abstract:}

\section{Background}

18 The research in the neuroscience field has evolved to use complex imaging and computational

19 tools to extract comprehensive information from data sets. Calcium imaging is a widely used

20 technique that requires sophisticated software to get precise and reproducible results. Many

21 laboratories struggle to adopt computational methods due to the lack of computational

22 knowledge and paywalls for software.

23 New Method

24 Here we propose a calcium imaging analysis method using TrackMate, an open-source Fiji plugin,

25 to track neurons at single-cell resolution, detect regions of interest (ROIs), and extract

26 fluorescence intensities. For confocal images, this method uses the maximal value to represent

27 the cell intensity for each z stack. This method can be done without coding or be combined with

28 Python or Jupyter Notebook scripts to accelerate the analysis.

29 Results

30 This method is validated in fly larval cool neurons, whose calcium changes in these neurons

31 respond to temperature fluctuation. It also identifies potential problems in approaches that

32 extract signals from maximal projection images.

33 Comparison with existing methods

34 This method does not depend on programming knowledge and/or commercial software but uses

35 open-source software and requires no coding abilities. Since TrackMate automatically defines

36 ROIs, this method greatly avoids human error and increases reproducibility. In addition, practice 
37 images and a step-by-step guide are provided to help users adopt this method in their

38 experiments.

39 Conclusions

40 Therefore, this open-source method allows for the analysis of calcium imaging data at single-cell

41 resolution with high reproducibility and has the potential to be applied in various cell types and

42 animal models by researchers with different programming abilities. 


\section{Introduction:}

Intracellular calcium level is a precise marker for neuronal excitability. Calcium imaging

studies have increasingly used this method with the development of techniques for measuring

intracellular calcium concentration, including genetically encoded calcium indicators (GECl), such

as GCaMP [2], which can be expressed in specific sets of neurons noninvasively by genetic

approaches. Lower costs of lasers and microscope components have also increased the use of

calcium imaging [3]. Importantly, this method allows for recording and studying single neurons

or large neuron populations simultaneously in freely moving animals $[2,4,5]$.

However, the analysis of calcium imaging data is challenging because (1) it involves

54 intermittently disappears or reappears with neuronal responses; and (3) neurons may shift in all

55 directions, specifically in and out of the focal plane [3]. Many laboratories perform calcium

imaging analysis manually, which is a time-consuming process and becomes impractical as the

length of recordings and the number of neurons increases. Manual analysis also introduces

operator bias, is prone to error, and affects the replicability of experiments [5].

Various software has been developed by individual laboratories to accelerate the process

60 of calcium imaging and increase reproducibility. Previously, software was designed in a limited

61 experimental context making it difficult for other laboratories to adopt them [6]. Recent efforts

62 to meet modern standards for software sharing have led to the development of several tools to

63 analyze the calcium imaging data consistently across different groups [5-11]. Many of these tools

64 still require programming knowledge and/or depend on commercial software. Importantly, most 
65 of these tools focus on calcium imaging data that include hundreds or thousands of neurons. The

66 lack of programming knowledge and paywalls of software deter researchers from adopting these

67 tools, especially when they record sparse neurons that can be analyzed manually. Thus, there is

68 a need for an open-source method that is easy to understand, suitable to analyze less complex

69 calcium imaging data, accelerates the analysis process, and increases reproducibility. used in tracking particles in various biological studies involving live-cell imaging, including calcium

72 imaging [11-14]. Generally speaking, calcium imaging analysis includes three steps: motion

73 correction, region of interest (ROI) detection, and signal extraction [3, 5]. TrackMate can automatically perform all these three steps and thus it can significantly reduce operator error and increase reproducibility in calcium imaging analysis. sparse cells. This method is particularly apt to analyze confocal calcium imaging captured in high magnification so that each cell appears on more than one image in a z stack. Instead of using the maximal projection, the method extracts fluorescence signals from every image and uses the maximal value of each $z$ stack to represent the cell intensity [15]. Additionally, TrackMate

81 automatically tracks cell motion and defines the ROI, which significantly reduces human error 82 and increases reproducibility. This method does not require computing knowledge as every step

83 can be performed manually. To accelerate analysis, Python and Jupyter Notebook scripts and a 84 step-by-step guide are provided. We also include practice images to help new coders to 85 understand how to use these scripts [6]. Last but not least, this method is compatible with 86 standard laptop and/or desktop computers. 


\section{Materials and methods:}

$88 \quad 2.1$ Fly strains

Ir21a-Gal4 [16] and UAS-GCaMP6m (P\{20XUAS-IVS-GCaMP6m\}attp2) [17] were

90 previously described.

\section{$91 \quad 2.2$ Calcium imaging} current method. Briefly, in fly larvae, dorsal organ cool neurons expressed the calcium indicator,

GCaMP6 [17], by Ir21a-Gal4 [16]. Three-day-old larvae were immobilized between a glass slide

60 seconds and returned to about $27^{\circ} \mathrm{C}$ for 30 seconds.

98

\subsection{Statistical analysis}

Statistical details of experiments are mentioned in the legend of Fig 3D-3G. The normality of distributions was assessed by the Shapiro-Wilk W test ( $p \leq 0.05$ rejected normal distribution). Statistical comparisons of normally distributed data were performed by Welch's t test or paired t test. For data that did not conform to a normal distribution, statistical comparisons were performed by the Wilcoxon test. Data analysis was performed using GraphPad Prism 9.

\subsection{Data and code availability}

Python and Jupyter Notebook scripts, as well as practice folders, have been deposited in GitHub and can be accessed at: https://github.com/niflylab/CalciumlmagingAnalysis. 


\section{Results:}

110 files and organize them. Step 2 is to identify z positions including cells of interest. Step 3 is to use

111 TrackMate to track cells and extract their intensities. Step 4 is to choose the maximal value at

112 each time point and use it to represent the cell intensity. Further analysis, such as to calculate

$113 \Delta \mathrm{F} / \mathrm{F}_{0}$, may be necessary. While all steps can be done manually, Python and Jupyter Notebook

114 scripts are provided for Step 1 and Step 4 to accelerate analysis.

\section{$115 \quad 3.1$ Organize .tif files}

For the convenience of the following steps, we recommend exporting calcium imaging

117 files to .tif files. A folder titled Practice 1 is provided for practice purposes on GitHub. We

118 recommend using the name of the calcium imaging file to name this folder. For example, if the

119 name of the calcium imaging file is NeuronOto2.czi (.czi is the file extension of Zeiss images), the

120 folder name should be Neuron0to2. Within the Practice 1 folder, there is a folder titled

121 Neuron0to2 that contains 21 images (Fig 2A). The format of their names is

122 Neuron0to2_h\#t\#z\#c\#.tif. $h$ is the phase. The number after $h$ is the phase number. One phase is

123 analyzed at a time. All images in this folder were taken in phase $01 . t$ is the time point or the $z$

124 stack. Twenty-one images in Fig 2A were taken at 3 time points: 01, 02, and 03. z represents the

$125 \mathrm{z}$ position. Each time point contains $7 \mathrm{z}$ positions: 01 to $07 . \mathrm{c}$ is the channel. Channel 2, $\mathrm{c} 2$, is used

126 to take these images. Of note, the example is a Zeiss format. Other confocal software may give 
127 different name formats. The images from the same $\mathrm{z}$ position are recommended to be saved in

128 the same folder (Fig 2B).

129 Next, the $z$ positions including cells of interest need to be identified manually. At each

130 time point, a single cell always appears in multiple $z$ positions (Fig 3A, 3B). All z positions

131 containing the cell should be analyzed. For the cell dictated by white arrowheads, z positions of

1325 to 8 should be analyzed. For the cells shown by orange and blue arrowheads, z positions of 8

133 to 13 should be analyzed. It is important to note that a z position may contain more than one cell

134 of interest (Fig 3B).

135 Since TrackMate requires grayscale images, these tif files need to be converted to 136 grayscale images. While Nikon NIS elements viewer and Leica LAS X export grayscale .tif files,

137 Zeiss ZEN lite exports color images. When necessary, we recommend using the Channels Tool in

138 Fiji (Image > Color > Channels Tool) to convert color images to grayscale images to avoid losing

139 the fluorescence information.

140 This process can be done by the Python or Jupyter Notebook script named

141 gray_sort_tiff_1, which is provided on GitHub.

\section{$142 \quad 3.2$ Extract the fluorescence intensity}

144 extraction of the fluorescence intensities of cells of interest [12, 14]. Fiji is an open-source image

145 processing program for analyzing scientific multidimensional images

146 (https://imagej.net/Fiji/Downloads). The folder titled Practice 2 on GitHub is provided for

147 practice. It is recommended to be downloaded onto the Desktop. In this folder, there are 7

148 folders named Neuron0to2_1 to Neuron0to2_7. These 7 folders represent 7 z positions. Each 
149 folder contains 3 images that are from the same z position but different time points (Fig 2B). Of

150 note, the cell dictated by white arrowheads in Fig 3B appears in z positions of 1 to 3 . So, the 151 images in folders of Neuron0to2_1 to Neuron0to2_3 are used to analyze this cell. The cells

152 dictated by orange and blue arrowheads in Fig 3B appear in z positions of 4 to 7 . So, images in

153 folders of Neuron0to2_4 to Neuron0to2_7 are used to analyze these two cells.

154 Fiji can open all images in a folder and present them as one stack in order. For example,

155 when the folder Neuron0to2_1 is opened, all images in this folder are opened in order as a stack.

156 Then, TrackMate is run. We recommend adjusting the following parameters in TrackMate.

157 1. Use the DoG detector. DoG detector works best for small spot sizes (Fig 4A).

158 2. Change the blob diameter, threshold, and median filter. Adjust the blob diameter based on

159 the sizes of cells. The blob diameter should be similar to the diameter of cells. If cells are oval,

160 the blob diameter should be similar to the minor axis. An increase of the threshold and use

161 of the median filter can help avoid background noise being picked up as signals. For the

162 practice images, we recommend setting the blob diameter to 22 , the threshold to 1 , and using

163 the median filter (Fig 4B).

164 3. When irrelevant signals are picked up, set filters can help remove some, if not all, irrelevant

165 signals. Filters $X$ and $Y$ can easily remove the irrelevant signals that are distant from the real

166 signals. When filters are set on one image, it is crucial to check all other images to make sure

167 the real signals are not removed. In practice imaging, there are three cells of interest and two

168 of them appear on the same z positions. We recommend analyzing them one by one. In Fig

$1694 \mathrm{C}$, to analyze the left cell, the right cell (arrowhead) and irrelevant signal (arrow) (Fig 4C1)

$170 \quad$ can be removed by setting filters $X$ and $Y$ (Fig 4C2). 
171 4. We recommend setting linking max distance and gap-closing max distance to be $3-5$ times

172 of the blob diameter, especially when samples are moving over time. This can help decrease

173 the number of tracks. We recommend setting gap-closing max frame gap to the number of

174 images in the stack. For the practice images, we recommend setting linking max distance and

175 gap-closing max distance to 110 and gap-closing max frame gap to 3 (Fig 4D).

176 5. To export the data, we recommend choosing the option of Export all spots statistics (Fig 4E)

177 and pressing the Execute button. The All Spots statsitics.csv is created. In this file, each row

178 shows the information of a ROI. Highlighting a ROI in the All Spots statistics window in Fiji

179 shows the corresponding ROI in green in the Fiji image. The same TRACK_ID is supposed to

180 represent the same ROI at different time points. However, this is not always true and needs

181 to be checked manually. For code users, we recommend setting a folder for each cell and

182 saving the All Spots statsitics.csv file as Mean_Intensity\#.csv (\#: the z position of the images)

183 in the corresponding folder.

184 In the All Spots statistics file, the column POSITION_T shows the time point and

185 MEAN_INTENSITY shows the mean intensity of the cell of interest. These values include

186 background. So, a background value needs to be extracted and subtracted from the

187 MEAN_INTENSITY. It is recommended to calculate the background intensity by using the

188 mean value of 3-5 nearby same size blobs that don't contain fluorescence signals and are

189 from different time points for each z position. Once subtracted, this difference is used to

190 represent the intensity of the cell of interest at this $z$ position for each time point. All of the $z$

191 positions that contain the cell of interest need to be analyzed. The maximal value at each 

to get $\Delta F / F_{0}$, can be performed manually.

194 6. If there are multiple cells of interest, repeat steps 1 to 5 to get the information of intensity change over time for each cell. scripts are provided for the following analysis.

\subsection{A step-by-step guide for new coders}

Notebook is .ipynb. For experienced Python coders, .py scripts are also provided on GitHub.

210 and Neuron 2 (orange and blue arrowheads in Fig 3B). Thus, there are 3 background values for

211 Neuron 0 and 4 background values for Neuron 1 and Neuron 2. Neuron 1 and Neuron 2 share

212 identical background values because they appear on the same z positions. Moreover, TrackMate

213 data have been saved in folders of Neuron 0, Neuron 1, and Neuron 2. The name of the All Spots 
214 statistics.csv files exported from Fiji needs to be changed to Mean_Intensity\#.csv and saved in

215 corresponding folders. \# is the number of the $z$ position. For example, in the Neuron 0 folder,

216 there are 3 .csv files named Mean_Intensity01, Mean_Intensity02, and Mean_Intensity03. They

217 are Neuron 0's All Spots statistics.csv in z position 1 to 3, respectively. Similarly, in folders of

218 Neuron 1 and Neuron 2, there are 4 .csv files, each containing information for Neuron 1 and

219 Neuron 2 extracted by TrackMate from z positions 4 to 7.

220 In the Neuron0to2 folder, there are 21 .tif files. These images are from 3 time points

221 (position_t) and each time point contains 7 z positions (position_z).

\section{$222 \quad$ 3.3.1 Preparation}

223 To run the script, the following files and software must be downloaded and/or installed.

224 1. Download the .ipynb files from GitHub and save them on the Desktop.

225

2. Download and install Individual Edition Anaconda from

https://www.anaconda.com/products/individual.

3. Open Anaconda-Navigator and launch Spyder.

The screen in Spyder contains 3 parts. The bottom right corner is the iPython console. In the iPython console. 
Use the following code line to install imagecodecs.

The system will check the existence of these packages before installing. Thus, this step

will not pose any problems even the packages have already been installed.

4. Quit Spyder and launch Jupyter Notebook from Anaconda-Navigator.

This file is used to convert color images to grayscale images, if they are not grayscale, and the Desktop. In this file, there are three boxes.

Box 1 functions to import os, cv2, and tifffile. It should not be edited.

Box 2 functions to convert the calcium imaging .tif files to grayscale files and save them

251 in proper folders. If the original files are grayscale, they will be copied and sorted into proper

252 folders. The original files will not be changed. If the calcium imaging is obtained from a Zeiss

253 confocal, box 2 may not need to be edited (but, we still recommend checking the file name format

254 and, if necessary, change box 2 as described below). If the calcium imaging is obtained from a

255 confocal other than Zeiss, please change the following code line based on the information in the

256 paper. This code line appears 4 times in the box 2 and all 4 lines must be changed. 
file $=f^{\prime}\left\{f i l e \_n a m e\right\} \_\{\text {phase }\} t\{t\} z\{z\}\{$ channel $\} . t i f '$

This code line includes 5 variables for the name of the file. \{file_name\} is the file name and should not be edited. _ is automatically added by the Zeiss software when converting the

261 calcium imaging to .tif files. \{phase\} is phase. If the name has no phase, 'Na' should be input in

262 box 3 and it will be skipped. Of note, it must not be deleted. $\{t\}$ is t position of the image. $\{z\}$ is $z$ position. Each image has a unique combination of $t$ position and $z$ position. \{channel\} is channel.

264 If the name does not include channel, ' $N a$ ' should be input in box 3 and it will be skipped.

For example, if the name of the calcium imaging is Neuron0to2 and the name of .tif files are

Neuron0to2Z\#T\#.tif, all 4 lines would be changed to the following:

file $=f^{\prime}\{$ file_name $\}\{$ phase $\} Z\{z\} T\{t\}\{$ channel $\}$. tif'

The letters in the curly brackets following the $\mathrm{Z}$ and $\mathrm{T}$ are also changed to match their

271 variables defined by the original script. In addition, phase and channel must not be deleted but 272 set ' $\mathrm{Na}$ ' in box 3.

Box 3 is used to input the information of the calcium imaging .tif files. The following

274 information is needed: (1) The location of the folder in which the calcium imaging tif files are 275 saved. The location can be found by dragging the folder to a browser search bar, such as Google

276 Chrome and Firefox. (2) The phase to be analyzed. (3) The number of time points (position_t). (4)

277 The number of z positions (position_z). (5) The channel to be analyzed. (6) Whether the files are 278 grayscale. The following box is box 3 from a Mac computer. 
phase $=$ 'h01',

position_t $=3$,

is_gray = 'No'

The following box is box 3 from a Windows computer.

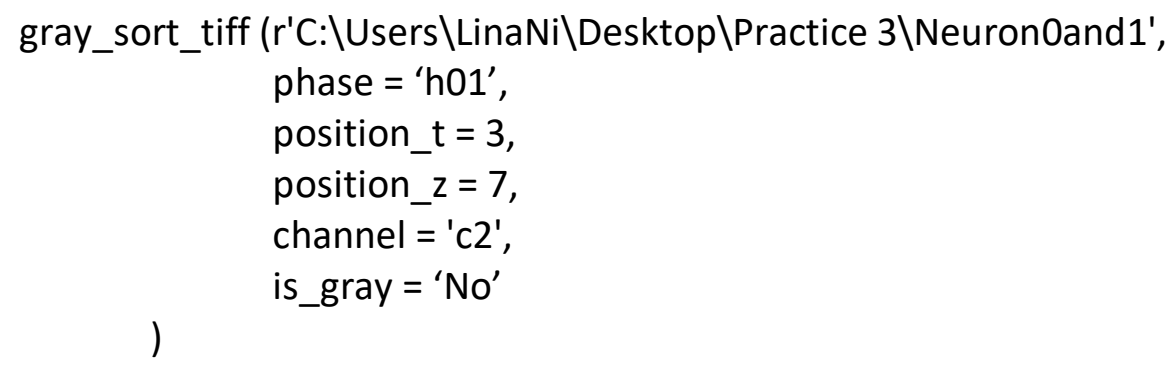

In line 1, input the location of the folder between single quotation marks. Quotation marks must not be deleted or added. On Mac computers, the file location is: /Users/LinaNi/Desktop/Practice 3/Neuron0and1. On Windows computers, a C: needs to be added at the beginning and thus it is: C: \Users\LinaNi\Desktop\Practice 3\Neuron0and1. The file location must be identified on the computer that is used to run the script. So, the example of the file location only works on the author's computer and will cause errors on other computers. Any slash (/) or backslash ( $($ ) at the end of the file location must be deleted.

In line 2 , input the phase name that is to be analyzed. In the example, the phase is 'h01'. If the file name does not include the information of the phase, input ' $\mathrm{Na}$ '. Of note, it is case sensitive. Thus, the input of 'na', 'NA', or ' $\mathrm{H} 01$ ' will cause an error. The quotation marks are necessary.

In line 3, input the number of time points (position_t) after the equal sign. In the example, the number is 3 . Since this is a number value, quotation marks are not needed. 
In line 4, input the number of z positions (position_z) after the equal sign. In the example,

310 the number is 7.

311 In line 5, input the channel name that is to be analyzed. If the file name has no information

312 about the channel, input ' $N a$ '. In the example, the channel is ' $c 2$ '.

313 In line 6, input whether the tif files are grayscale. If the tif files are grayscale, input 'Yes'.

314 If the tif files are not grayscale, enter 'No'.

315 To run this file, select box 1 and click the Run button in the menu bar. After it is done, box

3162 is automatically selected. Click Run to execute box 2. Then, box 3 is selected, and click Run.

317 In the Practice 3 folder, another folder named Neuron0to2_gray_stacks is created. In this

318 folder, there are 7 folders, each containing 3 grayscale images (Fig 5B). These 7 folders represent

319 the $7 \mathrm{z}$ positions. Within each folder, there are 3 images from the same $\mathrm{z}$ position but at different

320 time points.

Now the original calcium imaging tif files have been converted to grayscale images and

322 sorted into proper folders according to their z positions. They are ready to be analyzed by

323 TrackMate. To minimize modification of the following scripts, TrackMate data and background

324 intensities are recommended to be saved as shown in Fig 5A.

\subsubsection{Run individual_dFoverF0_1.ipynb}

This file is used to extract the intensities, calculate $\Delta F / F_{0}$ for individual cells at each time

327 point, and generate a plot to show the intensity change over time. individual_dFoverF0_1.ipynb

328 is recommended to be saved on the Desktop. In this file, there are three boxes. 
Mean_Intensity\#.csv files for each neuron. 2) It subtracts the background values from the

box is box 3 from a Mac computer.

loop_fluorescence_extract ( $r^{\prime}$ /Users/LinaNi/Desktop/Practice 3/Analysis',

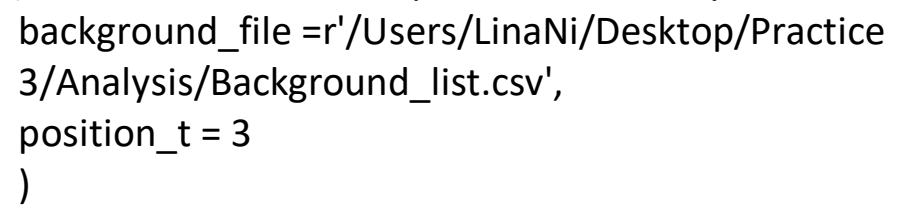
position_t $=3$ )

loop_fluorescence_extract ( $r^{\prime} C: \backslash U s e r s \backslash L i n a N i \backslash D e s k t o p \backslash$ Practice 3\Analysis', background_file $=r^{\prime} C: \backslash$ Users $\backslash$ LinaNi $\backslash$ Desktop $\backslash$ Practice 3\Analysis\Background_list.csv', The following box is box 3 from a Windows computer. position_t $=3$ ) 
3/Analysis/Background_list.csv. On a Windows computer, the file location is:

360 the number is 3.

Run boxes 1 to 3 one by one. positions is combined and saved (Fig 5C). In the Analysis folder, a folder named results is created.

\subsubsection{Run merge_dFoverF0_1.ipynb}

This file is used to combine the $\Delta F / F_{0}$ information of all cells, calculate the average and 
378 location of the results folder. (2) The number of time points (position_t). The following box is box

3793 from a Mac computer.

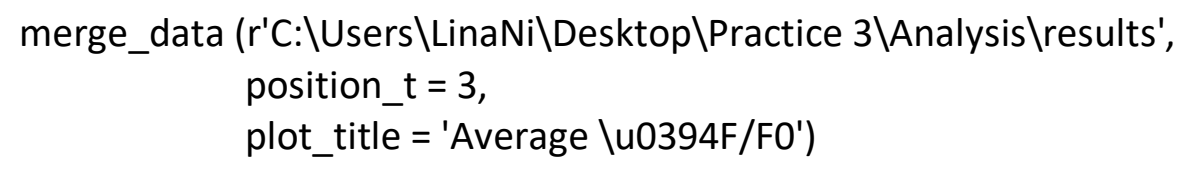

In line 1, input the location of the results folder between two single quotation marks. On a Mac computer, the file location is: /Users/LinaNi/Desktop/Practice 3/Analysis/results. On a Windows computer, the file location is: C: UUsers\LinaNi|Desktop\Practice 3\Analysis\results. In line 2, input the number of time points (position_t) after the equal sign. In the example, the number is 3 .

Line 3 should not be edited.

Run boxes 1 to 3 one by one.

In the folder of results, another folder named merged_data is generated (Fig 5D). This folder contains a .csv file that include the $\Delta F / F_{0}$ information of all cells, the average $\Delta F / F_{0}$ and SEM at each time point, as well as a plot to show the change of mean $\Delta \mathrm{F} / \mathrm{F}_{0}$ over time.

\subsection{Fly larval cool neurons respond to temperature changes.}

The calcium changes in fly larval cool neurons responding to temperature fluctuation is used to validate the method. A genetically encoded calcium indicator, GCaMP6m [17], was expressed in larval cool neurons by Ir21a-Gal4 [16]. When exposed to about $27^{\circ} \mathrm{C}$, the neurons 
403 had a low intracellular calcium level (Fig 3A, 3C, and 3D). When the temperature was decreased

404 to about $10^{\circ} \mathrm{C}$ and held, the intracellular calcium level was greatly increased and sustained (Fig $4053 \mathrm{~B}, 3 \mathrm{C}$, and 3D). The calcium level rapidly dropped with the increase of temperature (Fig 3C). Of 406 note, a similar plot to Fig 3C can be generated by the Python or Jupyter Notebook Scripts. In the previous study, maximal projection images were used to extract the fluorescence

408 intensities [18]. Though the trends of $\Delta F / F_{0}$ over time were similar, the plateau was higher from 409 maximal projection images. To understand the cause of this difference, we compared 410 background intensities that were used in both two studies. Background intensities from 411 individual z positions were significantly lower than that from maximal projection images (Fig 3E).

412 The cell intensities at the position_t $=11$ and position_t $=31$ were also compared (Fig 3F, 3G). No

413 difference was observed at the position_t $=11$ when cool cells were exposed at about $27^{\circ} \mathrm{C}$ and 414 they were inactive (Fig 3F). When cool cells were exposed at about $10^{\circ} \mathrm{C}$ at the position_t $=31$, 415 cell intensities from individual $z$ positions were significantly higher than that from maximal 416 projection images (Fig 3G). These data suggest that extraction of fluorescence signals from 417 maximal projection images may overestimate the background intensities and/or underestimate 418 cell signals, both of which affect $\Delta \mathrm{F} / \mathrm{F}_{0}$.

\section{Discussion:}

421 confocal calcium imaging with one or a few cells of interest. This method is particularly apt for 422 calcium imaging that is captured using high magnification and most cells appear on several z 423 positions. In addition, Python and Jupyter Notebook scripts are provided to accelerate analysis. 424 A step-by-step guide and practice files are also included for new coders. Since Fiji, Python, and 
Jupyter Notebook are all open-source software, this method is completely free. Moreover, it is compatible with standard laptop and desktop computers.

429 calcium imaging. This manual process is time consuming and prone to human bias and error.

430 TrackMate achieves automation of motion correction, ROI identification, and data extraction. For

431 motion correction, TrackMate is used to track the movement in the lateral $(\mathrm{X}-\mathrm{Y})$ direction. During

432 image acquisition in a live organism, such movement is unavoidable even when it is immobilized.

433 In addition, some stimuli, such as temperature change, cause significant $z$ drifting. Increasing the

434 height of $z$ stacks can resolve this issue because it includes the cells of interest in the imaging. To

435 track the changes in the $z$ direction, all $z$ positions that include the cells of interest need to be

436 analyzed and the maximal values are used to represent the signal intensities at corresponding

437 time points. Importantly, when using the same parameter settings, fluorescence extraction by

438 TrackMate is consistent from one computer or operator to another and therefore increases

439 reproducibility. Another challenge for calcium analysis is that cells disappear without stimulation

440 and reappear with stimulation. TrackMate can track the cells when they reappear.

441 However, this is not a bias-free method. We realize two steps may introduce variations.

442 (1) The background intensities may cause variation. Operators are unlikely to pick the same

443 region to extract background intensities. To minimize the variation, we recommend extracting

444 background intensities using a nearby same size blob that does not contain any fluorescent cells.

445 Another recommendation is to use the average of 3-5 background intensities from different time

446 points and using the mean value as the background intensity for the corresponding z position. (2) 
447 Weak signals may also introduce variation. When the signals are weak, TrackMate may not be

448 able to recognize the cells of interest or may mistake noise as the signals of the cells. Researchers

449 have to manually decide whether these signals are correct.

$450 \quad$ In summary, this paper provides an open-source analysis method for calcium imaging that

451 is captured using high magnification and most cells appear in several z positions. It will help

452 researchers or laboratories who have limited or no programming knowledge to accelerate the

453 analysis process and increase reproducibility.

\section{Acknowledgements:}

Zeiss LSM 880 in the Fralin Imaging Center was used for collecting calcium imaging data.

456 We acknowledge Steven Giavasis for comments on the GitHub README file. This work was

457 supported by $\mathrm{NIH}$ R21MH122987 (https://www.nimh.nih.gov/index.shtml), and NIH

458 R01GM140130 (https://www.nigms.nih.gov/) to L.N. The funders had no role in study design,

459 data collection and analysis, decision to publish, or preparation of the manuscript. 


\section{References:}

461 1. Grienberger C, Konnerth A. Imaging calcium in neurons. Neuron. 2012;73(5):862-85.

462 2. Nakai J, Ohkura M, Imoto K. A high signal-to-noise $\mathrm{Ca}(2+)$ probe composed of a single 463 green fluorescent protein. Nat Biotechnol. 2001;19(2):137-41.

$464 \quad 3 . \quad$ Robbins MC, CN

465 Kaminski, CF. Calcium imaging analysis - how far have we come? F1000Research. 2021;10:258.

466 4. Oh J, Lee C, Kaang BK. Imaging and analysis of genetically encoded calcium indicators 467 linking neural circuits and behaviors. Korean J Physiol Pharmacol. 2019;23(4):237-49.

468 5. Cantu DA, Wang B, Gongwer MW, He CX, Goel A, Suresh A, et al. EZcalcium: Open-Source 469 Toolbox for Analysis of Calcium Imaging Data. Front Neural Circuits. 2020;14:25.

470 6. Eglen SJ, Marwick B, Halchenko YO, Hanke M, Sufi S, Gleeson P, et al. Toward standard 471 practices for sharing computer code and programs in neuroscience. Nature neuroscience. 472 2017;20(6):770-3.

473 7. Pnevmatikakis EA, Giovannucci A. NoRMCorre: An online algorithm for piecewise rigid 474 motion correction of calcium imaging data. J Neurosci Methods. 2017;291:83-94.

475 8. Nguyen JP, Linder AN, Plummer GS, Shaevitz JW, Leifer AM. Automatically tracking 476 neurons in a moving and deforming brain. PLoS Comput Biol. 2017;13(5):e1005517.

477 9. Lagache T, Hanson A, Pérez-Ortega JE, Fairhall A, Yuste R. EMC <sup $>2</$ sup $>$ : A versatile 478 algorithm for robust tracking of calcium dynamics from individual neurons in behaving animals. 479 bioRxiv. 2021:2020.06.22.165696.

480 10. Giovannucci A, Friedrich J, Gunn P, Kalfon J, Brown BL, Koay SA, et al. CalmAn an open 481 source tool for scalable calcium imaging data analysis. eLife. 2019;8.

482 11. Delestro F, Scheunemann L, Pedrazzani M, Tchenio P, Preat T, Genovesio A. In vivo large483 scale analysis of Drosophila neuronal calcium traces by automated tracking of single somata. 484 Scientific reports. 2020;10(1):7153.

485 12. Tinevez JY, Perry N, Schindelin J, Hoopes GM, Reynolds GD, Laplantine E, et al. TrackMate: 486 An open and extensible platform for single-particle tracking. Methods (San Diego, Calif). $487 \quad 2017 ; 115: 80-90$.

488 13. Schindelin J, Arganda-Carreras I, Frise E, Kaynig V, Longair M, Pietzsch T, et al. Fiji: an 489 open-source platform for biological-image analysis. Nat Methods. 2012;9(7):676-82.

490 14. Fazeli E, Roy NH, Follain G, Laine RF, von Chamier L, Hänninen PE, et al. Automated cell 491 tracking using StarDist and TrackMate. F1000Research. 2020;9:1279.

492 15. Dombeck DA, Khabbaz AN, Collman F, Adelman TL, Tank DW. Imaging large-scale neural 493 activity with cellular resolution in awake, mobile mice. Neuron. 2007;56(1):43-57.

494 16. Ni L, Klein M, Svec KV, Budelli G, Chang EC, Ferrer AJ, et al. The lonotropic Receptors IR21a 495 and IR25a mediate cool sensing in Drosophila. eLife. 2016;5.

496 17. Chen TW, Wardill TJ, Sun Y, Pulver SR, Renninger SL, Baohan A, et al. Ultrasensitive 497 fluorescent proteins for imaging neuronal activity. Nature. 2013;499(7458):295-300.

498 18. Tyrrell JJ, Wilbourne JT, Omelchenko AA, Yoon J, Ni L. lonotropic Receptor-dependent cool 499 cells control the transition of temperature preference in Drosophila larvae. PLoS genetics. $500 \quad 2021 ; 17(4): \mathrm{e} 1009499$. 


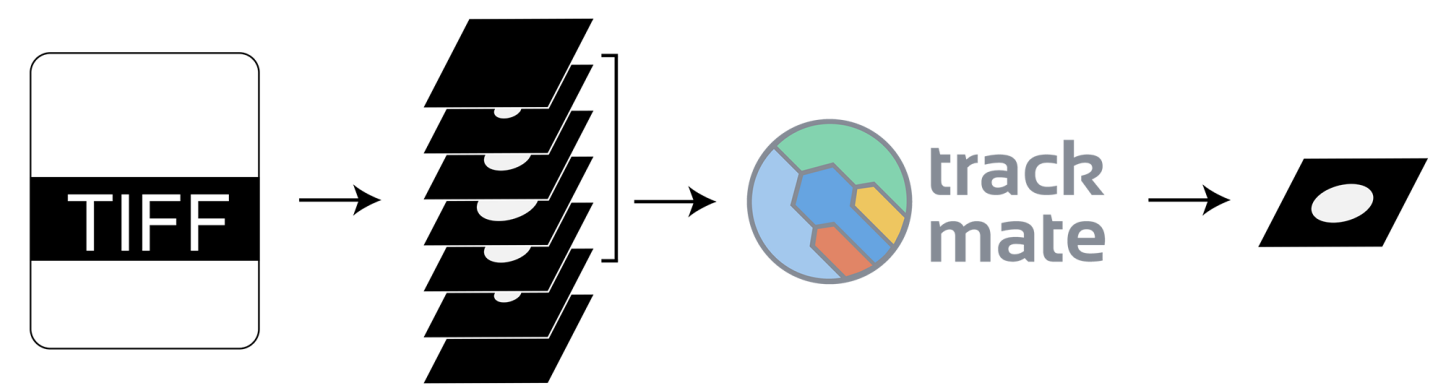

504 Fig 1. Workflow of confocal calcium imaging analysis with sparse cells. This analysis includes

Step 1. Convert confocal images to .tif files
Step 2. Identify z positions including cells of interest in each time point
Step 3. Use Trackmate to extract the cells' intensities on each z position over time
Step 4. Use the max intensity to represent a cell's intensity at each time point four steps. The first step is to convert confocal images to .tif files. This step can be done by confocal software or Fiji. Images from the same z position over time are recommended to be saved in the same folder for analysis. The second step is to identify z positions including cells of interest at each time point. At each time point, a cell usually appears on multiple z positions; different cells may appear on the same or different $z$ positions. Only the $z$ positions that include cells of interest need to be analyzed. The third step is to use TrackMate, a plugin in Fiji, to extract cells' intensities in each image. Images from the same z position over time are recommended to be analyzed as a stack. All z positions including cells of interest need to be analyzed. The fourth step is to determine the maximal intensity of each cell at each time point and use it to represent the intensity of this cell at this time point. All steps can be done manually. Python and Jupyter 
517 Figure 2

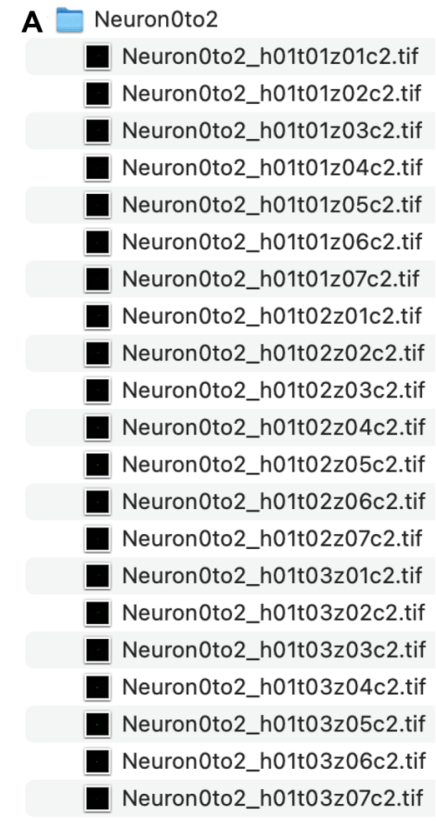
of the $z$ position.
B $\square$ Neuron0to2_1

Neuron0to2_h01t01z01c2.tif

Neuron0to2_h01t02z01c2.tif

I NeuronOto2_h01t03z01c2.tif

$\checkmark \square$ Neuron0to2_2

- Neuron0to2_h01t01z02c2.tif

Neuron0to2_h01t02z02c2.tif

Neuron0to2_h01t03z02c2.tif

$\checkmark$ Neuron0to2_3

I Neuron0to2_h01t01z03c2.tif

Neuron0to2_h01t02z03c2.tif

Neuron0to2_h01t03z03c2.tif

$\checkmark \square$ Neuron0to2_4

Neuron0to2_h01t01z04c2.tif

Neuron0to2_h01t02z04c2.tif

Neuron0to2_h01t03z04c2.tif

$\checkmark$ Neuron0to2_5

I Neuron0to2_h01t01z05c2.tif

Neuron0to2_h01t02z05c2.tif

Neuron0to2_h01t03z05c2.tif

$\checkmark \square$ Neuron0to2_6

Neuron0to2_h01t01z06c2.tif

Neuron0to2_h01t02z06c2.tif

Neuron0to2_h01t03z06c2.tif

$\checkmark \square$ Neuron0to2_7

Neuron0to2_h01t01z07c2.tif

Neuron0to2_h01t02z07c2.tif

Neuron0to2_h01t03z07c2.tif

Fig 2. An example of the organization of the tif files. (A) The folder name must be the same as the name of the calcium imaging. Neuron0to2 is the name of calcium imaging file and _h\#t\#z\#c\# is the specific information of each .tif file. (B) The images from the same $z$ position must be saved in the same folder. The folder name is the name of calcium imaging file followed by the number 

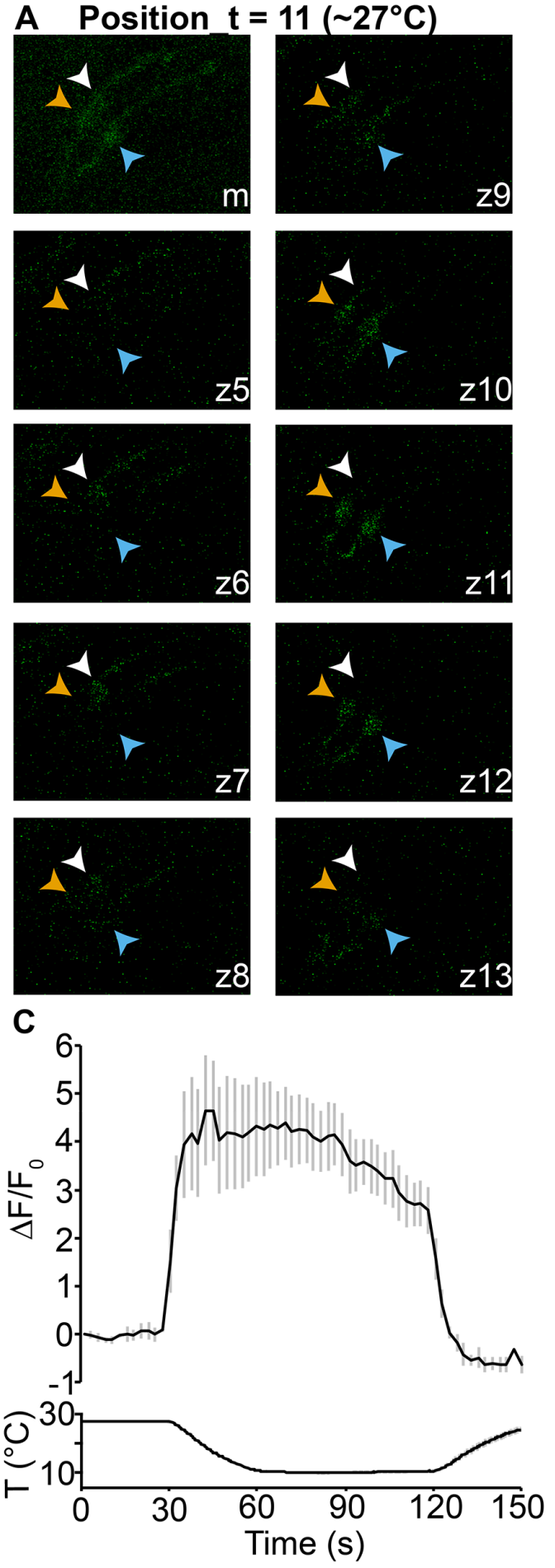
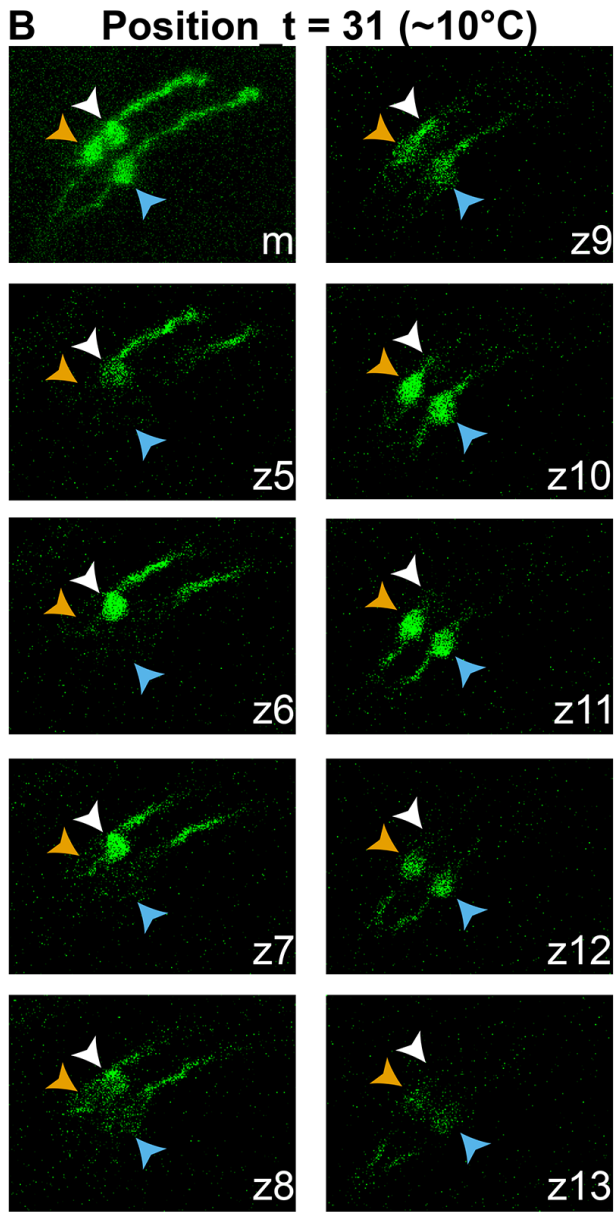

D
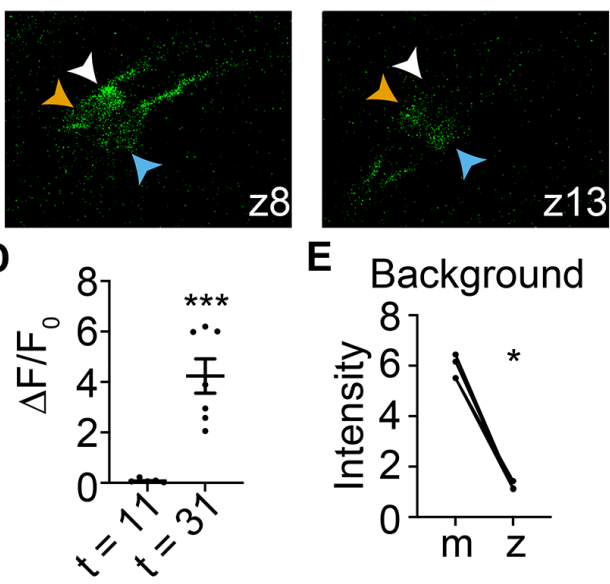

E Background
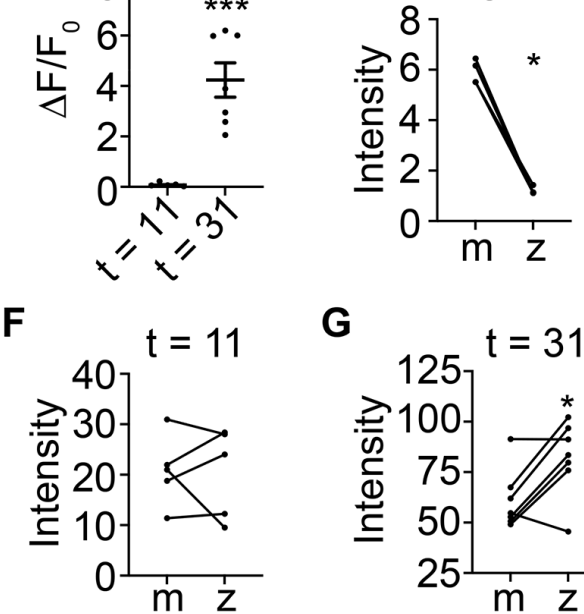

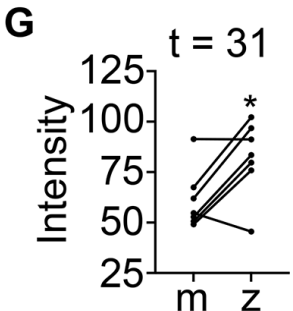

Fig 3. Calcium imaging of fly larval cool cells in the inactive and active states. (A) Cells are barely visible in the inactive state. (B) Cells are strongly fluorescent in the active state. Different color arrowheads dictate different cells. m: maximal projection. z5-13: z positions from 5 to 13 . In (B), 
530 the cell dictated by white arrowheads is shown on $z 5$ to $z 8$; the cells dictated by orange and blue 531 arrowheads are shown on $z 8$ to $z 13$. (C) Fluorescence is quantified as the change in fluorescence 532 intensity compared to the initial intensity. $n=7$ cells from 3 animals. Traces, mean \pm SEM. (D) The 533 changes in fluorescence intensities compared to the initial intensity at indicated time points. 534 Welch's t test, $* * * p<0.001$. (E) Background intensities from maximal projection images $(\mathrm{m})$ and 535 from individual z positions (z). Wilcoxon test, $* p<0.05$. (F, G) Fluorescence intensities from 536 maximal projection images $(\mathrm{m})$ and from individual $\mathrm{z}$ positions $(\mathrm{z})$ at indicated time points. Paired 537 t test, $* p<0.05$.

538 

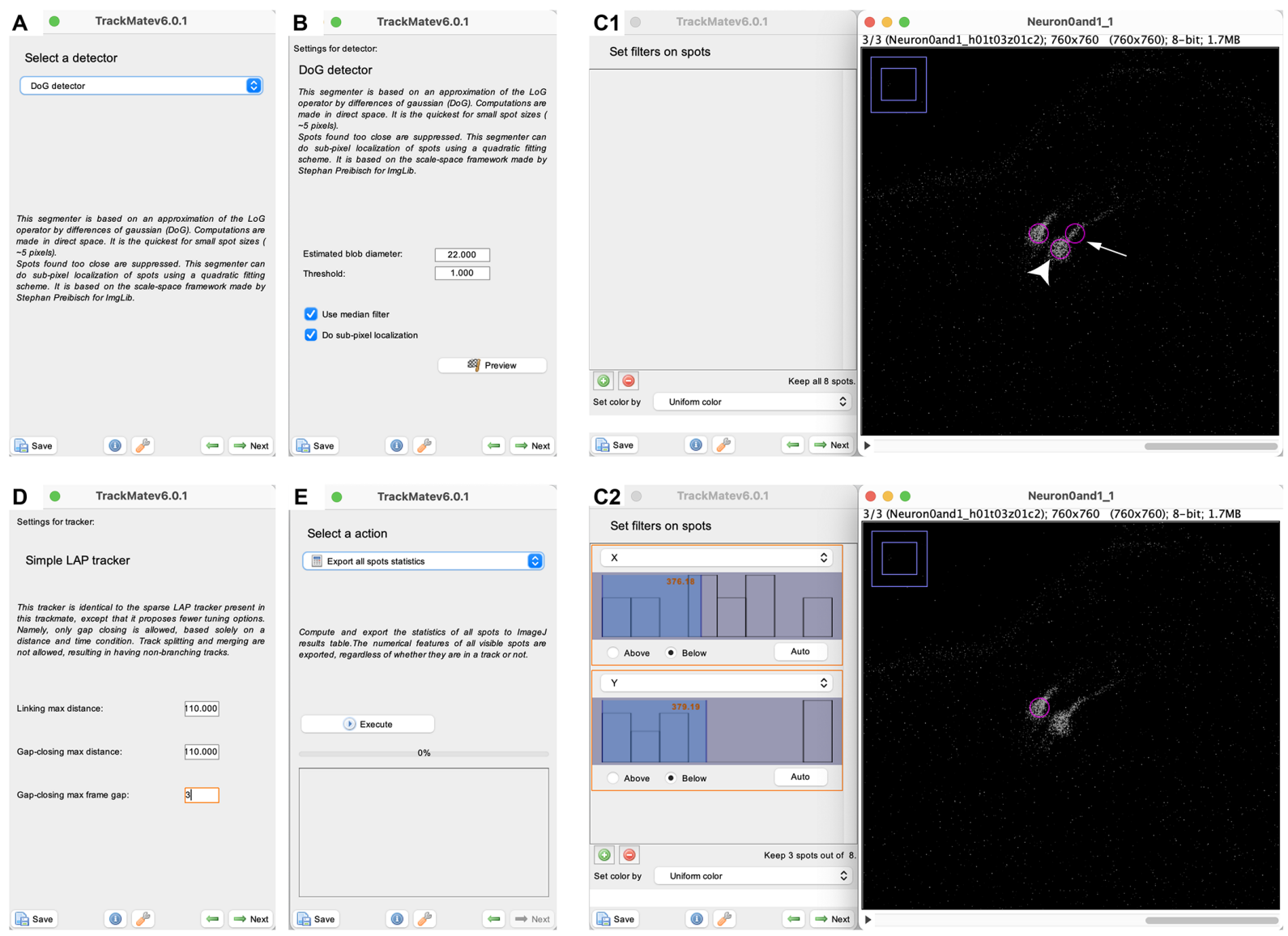

Fig 4. Use Trackmate, a Fiji plugin, to extract cells' intensities. The following parameters are recommended to be input. (A) Select a detector. We recommend using the DoG detector that works best for small spot sizes. (B) DoG detector. We recommend changing the blob diameter based on the sizes of cells. The blob diameter should be similar to the diameter of cells. If cells are oval, the blob diameter should be similar to the minor axis. Increasing the threshold can help decrease the effects of the background noise. If signals are strong, we recommend using the median filter that can help decrease the Salt and Pepper noise. (C) Set filters on spots. When irrelevant signals are picked up, filters can help remove some, if not all, irrelevant signals. Filters $X$ and $Y$ can easily remove the irrelevant signals that are distant from the real signals. When filters are set on one image, it is crucial to check all other images to make sure the real signals are not removed. In (C1), two cells of interest and one irrelevant signal (arrow) are picked up (magenta circles). To analyze the left cell, the right cell (arrowhead) and irrelevant signal (arrow) can be removed by setting filters $X$ and $Y(C 2)$. (D) Simple LAP tracker. We recommend setting linking max distance and gap-closing max distance to be $3-5$ times of the blob diameter, especially when samples are moving over time. This can help decrease the number of tracks. We recommend setting gap-closing max frame gap to the number of images in the folder. (E) Select an action. We recommend choosing the option of Export all spots statistics. 
Figure 5

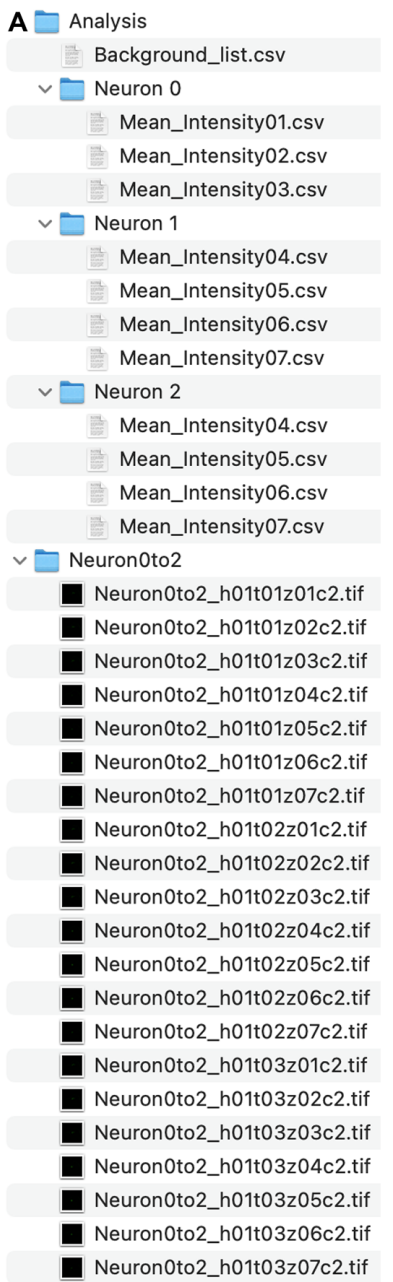

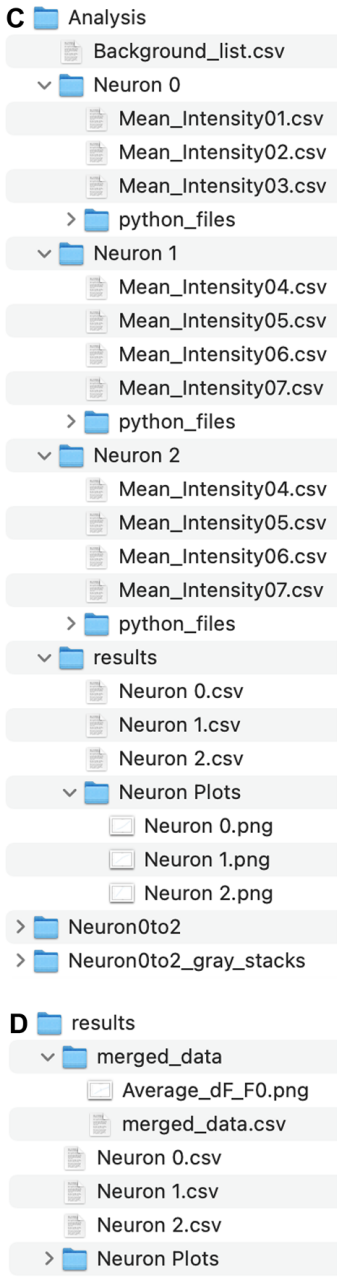

Fig 5. The information in the folder of Practice 3 before and after analysis using Jupyter Notebook scripts. (A) Before analysis, in the folder of Practice 3, there is an Analysis folder and a Neuron0to2 folder. In the Analysis folder, there is a file named Background_list.csv and three folders named Neuron 0, Neuron 1, and Neuron 2. The Background_list.csv includes the background intensities of Neuron 0, Neuron 1, and Neuron 2 in different z positions. The Neuron 0 folder contains three .csv files that represent 3 z positions. In folders of Neuron 1 and Neuron 2 , each contains four .csv files that represent $4 \mathrm{z}$ positions. Each .csv file contains intensity information of the corresponding neurons from the same $z$ position. (B) After running gray_sort_tiff_1.ipynb, a folder named Neuron0to2_gray_stacks is created. In this folder, there are 7 folders, each containing 3 grayscale images. These 7 folders represent 7 z positions. In each folder, there are 3 images from the same $z$ position. (C) After running individual_dFoverF0_1.ipynb, in every of the Neuron 0, Neuron 1, and Neuron 2 folders, a folder named python_files is created. The intensity information of the corresponding neuron in different $z$ positions is combined and saved in this folder. In the Analysis folder, a folder named results is created. In this folder, the intensity information and plots for Neuron 0 , Neuron 1 , and Neuron 2 can be found. (D) After running merge_dFoverF0_1.ipynb, a folder named merged_data is generated in the results folder. This folder contains a .csv file, including the $\Delta F / F_{0}$ 
bioRxiv preprint doi: https://doi.org/10.1101/2021.09.28.462182; this version posted September 30, 2021. The copyright holder for this preprint (which was not certified by peer review) is the author/funder. All rights reserved. No reuse allowed without permission.

578 information of all cells, the average $\Delta F / F_{0}$ and SEM at each time point, and a plot to show the 579 change of mean $\Delta \mathrm{F} / \mathrm{F}_{0}$ over time. 